\title{
Role of dietary polyamines in a phase III clinical trial of difluoromethylornithine (DFMO) and sulindac for prevention of sporadic colorectal adenomas
}

\begin{abstract}
K P Raj ${ }^{1,2}$, J A Zell ${ }^{*}, 1,2,3$, C L Rock ${ }^{4}$, C E McLaren ${ }^{1,3}$, C Zoumas-Morse ${ }^{4}$, E W Gerner ${ }^{5,6}$ and F L Meyskens ${ }^{1,2}$
${ }^{1}$ Chao Family Comprehensive Cancer Center, University of California, Irvine, CA 92697, USA; ${ }^{2}$ Department of Medicine, University of California, Irvine, CA 92697, USA; ${ }^{3}$ Department of Epidemiology, University of California, Irvine, CA 92697, USA; ${ }^{4}$ Department of Family and Preventive Medicine, Cancer Prevention and Control Program, University of California, La Jolla, San Diego, CA 92093, USA; ${ }^{5}$ Department of Cell Biology and Anatomy, College of Medicine, Arizona Cancer Center, Tucson, AZ 85724, USA and ${ }^{6}$ Gastrointestinal Cancer Program, Arizona Cancer Center, Tucson, AZ 85724, USA
\end{abstract}

Background: The polyamine-inhibitory regimen difluoromethylornithine (DFMO) + sulindac has marked efficacy in preventing metachronous colorectal adenomas. Polyamines are synthesised endogenously and obtained from dietary sources. Here we investigate dietary polyamine intake and outcomes in the DFMO + sulindac colorectal adenoma prevention trial.

Methods: Dietary polyamine data were available for 188 of 267 patients completing the study. Total dietary polyamine content was derived by the sum of dietary putrescine, spermine and spermidine values and categorised into two groups: highest $(>75-100 \%)$ vs the lower three quartiles (0-25, 25-50 and 50-75\%). Baseline tissue polyamine concentration and ODC1 genotype were determined. Logistic regression models were used for risk estimation.

Results: A significant interaction was detected between dietary polyamine group and treatment with regard to adenoma recurrence $(P=0.012)$. Significant metachronous adenoma risk reduction was observed after $D F M O+$ sulindac treatment in dietary polyamine quartiles $1-3$ (risk ratio (RR) $0.19 ; 95 \%$ confidence interval (Cl) $0.08-0.42 ; P<0.0001$ ) but not in quartile 4 (RR 1.51; $95 \% \mathrm{Cl} 0.53-4.29 ; P=0.44)$. However, a lower number of events in the placebo group within dietary quartile 4 confound the aforementioned risk estimates.

Conclusion: These preliminary findings reveal complex relationships between diet and therapeutic prevention, and they support further clinical trial-based investigations where the dietary intervention itself is controlled.

Colorectal cancer (CRC) is the second most common cause of cancer-related deaths in the United States (Siegel et al, 2011). Understanding key mechanisms that explain colorectal tumorigenesis should facilitate the development of new approaches to CRC prevention. Compelling experimental and epidemiological studies indicate that diet and nutritional factors are important in modulating the transformation of normal epithelium into carcinoma. Identifying dietary constituents with antitumour

\footnotetext{
${ }^{*}$ Correspondence: Dr JA Zell; E-mail: jzell@uci.edu

Presented in part at the 2010 Gastrointestinal Cancers Symposium, 22-24 January 2010, Orlando, FL, USA, and at the 2010 American Society of Clinical Oncology Annual Meeting, 4-8 June 2010, Chicago, IL, USA.
}

Received 29 August 2012; revised 11 December 2012; accepted 16 December 2012; published online 22 January 2013

(C) 2013 Cancer Research UK. All rights reserved 0007-0920/13 
activity and investigating their mechanism of action may lead to significant advances in cancer prevention, particularly for cancers within the colon, which are directly exposed to dietary constituents(Linsalata and Russo, 2008).

Polyamines are organic cations found in every living cell (Wallace, 1996). They are synthesised endogenously from the arginine-derived product ornithine, by the rate-limiting enzyme ornithine decarboxylase (ODC) (Gerner and Meyskens, 2004). Ornithine is converted by ODC to the diamine putrescine, which is sequentially converted into the polyamines spermidine and spermine by spermidine and spermine synthases; transport, catabolic and interconversion mechanisms for these amines have been described (Gerner and Meyskens, 2004). A link between polyamines and cancer has been established for several decades. In addition to the well-described series of biochemical, genetic and epigenetic alterations involved in colorectal carcinogenesis (Fearon and Vogelstein, 1990), elevated epithelial polyamine content has been consistently implicated in CRC carcinogenesis (Jass et al, 2002; Gerner and Meyskens, 2004). Overexpression of ODC in the rectal mucosa has been associated with CRC risk and found to be a potential biochemical marker of CRC proliferation (McGarrity et al, 1990; Wang et al, 1996; Brabender et al, 2001). Polyamines regulate oncogene expression and function through transcriptional and posttranscriptional processes (Tabib and Bachrach, 1999; Bachrach et al, 2001). Difluoromethylornithine (DFMO, eflornithine) irreversibly inhibits ODC, which results in decreased polyamine synthesis (Gerner and Meyskens, 2004). Difluoromethylornithine is the most widely studied example of a polyamine-metabolism inhibitor that suppresses cancer development in animal models (Meyskens and Gerner, 1999), and together with the non-steroidal anti-inflammatory drug (NSAID) sulindac, has been recently shown by members of our group to decrease the incidence of metachronous colorectal adenomas in human clinical trials after a 3-year intervention when compared with placebos (Meyskens, 2008). Hence, there is compelling evidence indicating that the polyamines have important roles in governing cell proliferation, survival and apoptosis, suggesting that decreases in polyamine content may inhibit the cancer process.

In addition to endogenous polyamine production, dietary polyamines and their metabolism by intestinal microorganisms have been shown to be major determinants of the total body polyamine pool (Zoumas-Morse et al, 2007). Notable dietary polyamines include spermine, spermidine and putrescine. Major contributors of spermine in the United States diet are ground meat, lunch meat, green peas, lasagna and pasta with meat sauce, peanut butter, peanuts and other nuts (Zoumas-Morse et al, 2007). Major contributors of spermidine in the US diet are green peas, cheese, lasagna and pasta with meat sauce, potatoes, burritos, tacos, tostadas and quesadillas (Zoumas-Morse et al, 2007). Major contributors of putrescine in the US diet are oranges and grapefruit and their juices, fresh tomatoes, bananas, beer and corn (Zoumas-Morse et al, 2007). Polyamine absorption occurs in the gut, and the various polyamines are metabolised in tissues under strict regulation of ODC (Thomas and Thomas, 2003). Despite extensive evidence about dietary polyamines and their role in colon carcinogenesis in pre-clinical models, there are no prior studies to evaluate whether dietary polyamines influence tissue polyamines or adenoma formation in humans. Also, murine familial adenomatous polyposis (FAP) models indicate that dietary polyamines decrease the anticarcinogenic activity of sulindac (Thomas and Thomas, 2003), suggesting an interaction between dietary polyamine intake and certain chemopreventive agents. Therefore, we examined whether dietary polyamine intake influenced baseline adenoma characteristics, baseline rectal tissue polyamine levels and the incidence of metachronous colorectal adenomas via interaction with polyamine-inhibitory treatment in study participants of the randomised, phase III colorectal adenoma prevention study
(Meyskens, 2008) of DFMO + sulindac vs placebo. Our primary study aim was to analyse the interaction between dietary polyamines and treatment with $\mathrm{DFMO}+$ sulindac on adenoma recurrence.

\section{MATERIALS AND METHODS}

Patient population and description of parent study. This study involves analysis of patient data and specimens from the multicenter colon adenoma prevention trial, as described elsewhere (Meyskens, 2008). Major inclusion criteria were patients age 40-80 years with a history of at least one colorectal adenoma at least $3 \mathrm{~mm}$ in size within 5 years of study entry. Subjects were ineligible if they had FAP, hereditary nonpolyposis CRC, inflammatory bowel disease or invasive cancer within the past 5 years. It originated as a randomised double-blind, placebo-controlled phase IIb trial of DFMO $500 \mathrm{mg}$ daily in combination with sulindac $150 \mathrm{mg}$ daily $v s$ placebo in patients with prior history of colorectal adenoma, and was later expanded in 2003 to a phase III clinical trial. Final analyses included aggregate data from all patients enrolled in the phase IIb and phase III clinical trials. Threehundred and seventy-five patients were randomised to receive treatment with either DFMO and sulindac or placebo. Stratification in the parent study was performed for study site and prior low-dose aspirin usage. Planned treatment duration was 36 months. Baseline and end-of-study endoscopies were performed, each with procurement of eight rectal mucosa tissue biopsies. Clinical data were collected at baseline interview and recorded in the study chart. ODC1 (rs2302615) genotyping was conducted on patient-derived genomic DNA using allele-specific TaqMan probes as described previously (Guo et al, 2000; Zell et al, 2010a). A food frequency questionnaire (FFQ) completed by the patients was collected at the initiation of the study. At the second interim analysis, the study was halted because the clinical efficacy end points were achieved, thus 267 patients completed the trial (Meyskens, 2008).

This study involved analysis of data and tissue from the phase III colorectal adenoma prevention parent trial (Meyskens, 2008). The parent study was approved after full committee review by the UC Irvine institutional review board (IRB protocol no. 2002-2261) and each of the local IRBs from the participating clinical study sites. All patients signed informed consent for inclusion into the trial, and specimen retrieval/analysis for research purposes.

Dietary polyamine intake. Dietary intakes of participants in this clinical trial were estimated with the Fred Hutchinson Cancer Research Center FFQ, and the analytic algorithms for this instrument are published elsewhere (Kristal et al., 1992; Schakel, 1997). To estimate dietary polyamines in the 370 foods listed in this FFQ, a polyamine food content database was developed and linked to the FFQ, for which the University of Minnesota Nutrition Coordinating Center Nutrient Database serves as the primary source of food content data, as described by Zoumas-Morse et al (2007). Values for spermine, spermidine and putrescine in individual food items were calculated and expressed as $\mathrm{nmolg}^{-1}$ (Zoumas-Morse et al, 2007). Dietary putrescine was the major contributor to total dietary polyamine intake. Total daily dietary polyamine content was derived by adding the dietary putrescine, spermine and spermidine and further categorised into quartiles, separately for the baseline cohort, and end-of-study cohort.

Tissue polyamine analysis. Rectal tissue polyamine content was determined as previously described (Boyle et al, 1992; Meyskens et al, 1998). Polyamine content was evaluated using three randomly selected rectal mucosal biopsy specimens from the eight specimens available from each participant at baseline. These methods measured putrescine, cadaverine, histamine, spermidine, 
spermine, and monoacetyl derivatives of putrescine, spermidine and spermine (Meyskens et al, 1998). Tumour-free rectal biopsies were collected, flushed with ice-cold saline and stored frozen at $-80{ }^{\circ} \mathrm{C}$. Samples were processed, then assayed for polyamine content by reverse-phase high-performance liquid chromatography with 1,7-diaminoheptane as an internal standard (Seiler and Knodgen, 1980). Protein content in each sample was determined using the BCA protein assay kit (Pierce, Rockford, IL, USA). Data were expressed as nmol polyamine per milligram protein.

Statistical analysis. Descriptive analysis of variables such as age, gender, ethnicity, prior aspirin use, adenoma location, size and histology was performed. A variable called 'advanced adenomas' was assigned to include polyps with villous or tubulovillous features, high-grade dysplasia or carcinoma in situ. Consistent with previously defined adenoma nomenclature (Uemura et al., 2010), a variable called 'high-risk adenomas' was created that takes into account adenoma size, histology and synchronous multiple adenomas. As such, adenomas with villous histology, high-grade dysplasia, size $>1 \mathrm{~cm}$ or multiple adenomas (three or more) were classified as high-risk adenomas. Because dietary and tissue polyamine data were not normally distributed, the non parametric Wilcoxon rank-sum test was used for comparisons of numeric data. The $\chi^{2}$-test for independence was used for comparisons of nominal data. Spearman's rank correlation coefficient $\left(r_{\mathrm{s}}\right)$ was used to assess the relationship between dietary polyamine intake at baseline and intakes of protein, animal-derived protein and arginine. A logistic regression model was used to calculate the recurrence risk of metachronous adenomas. Patients in polyamine intake quartiles 1,2 and 3 reflected similar clinical outcomes in the initial regression models; therefore, these three quartiles were collapsed into one group. The final model included presence of metachronous adenoma as the outcome variable, and predictors including dietary polyamine group, treatment with $\mathrm{DFMO}+$ sulindac ( $v s$ placebo), low-dose aspirin use ( $v s$ none), large adenoma diameter ( $\geqslant 1 \mathrm{~cm} v s<1 \mathrm{~cm}$ ), ODC1 genotype (via the dominant genetic model: GG vs GA/AA) and a term representing interaction between dietary polyamine group and treatment. Exploratory analyses were done using the final model with either additional adjustment for quartiles of total energy intake or tissue polyamine levels. Additional models were done separately for each dietary polyamine group, with the predictors: treatment, low-dose aspirin use, large adenoma diameter and ODC1 genotype, with the following outcome variables: adenoma recurrence and advanced adenoma recurrence. All statistical tests were two-sided, and all analyses were conducted using SAS 9.2 statistical software (SAS Inc., Cary, NC, USA).

\section{RESULTS}

Characteristics of the study population. Dietary polyamine data were available for 222 of 375 study patients at baseline (baseline cohort), and for 188 of 267 patients completing the end-of-study colonoscopy (end-of-study cohort). The clinical characteristics of the study population are shown in Table 1. Patient groups were similar in age, gender, race/ethnicity, aspirin use, number of prior adenomas, adenoma histology and adenoma location. Mean age of the study population was 60 years \pm 8 s.d., ranging from 47 to 75 years at the time of study entry. The majority of patients were males (74\%) compared with females (26\%). The majority of patients were white non-Hispanic (86\%), followed by Hispanics (5\%), African-Americans (4\%) and Asian-Americans (3\%). Low-dose aspirin use at study entry was reported by 93 patients (42\%), compared with 129 non-aspirin users (58\%). Ninety-three patients (42\%) had just one adenoma at baseline, and the total number of baseline adenomas ranged from 1 to 16 . The following
Table 1. Characteristics of the baseline and end-of-study participants ${ }^{a}$ \begin{tabular}{|l|c|c|}
\hline & $\begin{array}{c}\text { Baseline } \\
\mathbf{n = 2 2 2}\end{array}$ & $\begin{array}{c}\text { End-of-study } \\
\boldsymbol{n}=\mathbf{1 8 8}\end{array}$ \\
\hline Mean age at study entry (years, w/range) & $60.7(41-78)$ & $60.4(41-78)$ \\
\hline
\end{tabular} Gender

\begin{tabular}{|l|r|r|}
\hline Male & $164(74 \%)$ & $141(75 \%)$ \\
Female & $58(26 \%)$ & $47(25 \%)$ \\
\hline
\end{tabular}

Ethnicity

\begin{tabular}{|l|c|c|}
\hline Caucasian & $192(86 \%)$ & $160(85 \%)$ \\
Hispanic & $12(5 \%)$ & $12(6 \%)$ \\
Black & $8(4 \%)$ & $6(3 \%)$ \\
Asian & $7(3 \%)$ & $7(4 \%)$ \\
Other & $3(2 \%)$ & $3(2 \%)$ \\
\hline
\end{tabular}

Treatment assignment

\begin{tabular}{|l|l|l|}
\hline DFMO + sulindac & $112(50 \%)$ & $94(50 \%)$ \\
Placebo & $110(50 \%)$ & $94(50 \%)$
\end{tabular}

\section{Aspirin use}

\begin{tabular}{|l|l|l}
\hline Yes & $93(42 \%)$ & $73(39 \%)$ \\
\hline
\end{tabular}

No $129(58 \%) \quad 115(61 \%)$

Number of adenomas 2 $\geqslant 3$ (multiple adenomas) $^{\mathbf{b}}$ Missing $93(42 \%) \quad 81(43 \%)$ $52(23 \%) \quad 47(25 \%)$ $74(33 \%) \quad 57(30 \%)$ $3(1 \%) \quad 3(1 \%)$

\section{Adenoma location}

\begin{tabular}{|l|l|l|}
\hline Rectum & $43(19 \%)$ & $37(20 \%)$ \\
Left colon & $87(39 \%)$ & $75(40 \%)$ \\
Transverse colon & $31(14 \%)$ & $25(13 \%)$ \\
Right colon & $35(16 \%)$ & $30(16 \%)$ \\
Cecum & $26(12 \%)$ & $21(11 \%)$ \\
\hline
\end{tabular}

Adenoma size

\begin{tabular}{|l|l|l|}
\hline$<5 \mathrm{~mm}$ & $69(31 \%)$ & $55(29 \%)$ \\
$5-9 \mathrm{~mm}$ & $85(38 \%)$ & $75(40 \%)$ \\
$10-15 \mathrm{~mm}^{\mathrm{b}}$ & $47(21 \%)$ & $40(21 \%)$ \\
$>15 \mathrm{~mm}^{\mathrm{b}}$ & $21(10 \%)$ & $18(10 \%)$ \\
\hline
\end{tabular}

\section{Adenoma histology}

\begin{tabular}{|l|c|c|}
\hline Tubular adenoma & $170(77 \%)$ & $143(76 \%)$ \\
Adenoma - NOS & $13(6 \%)$ & $11(6 \%)$ \\
Tubulovillous $^{\text {b }}$ & $30(13 \%)$ & $25(13 \%)$ \\
Villous $^{\text {b }}$ & $6(3 \%)$ & $6(3 \%)$ \\
High-grade dysplasiab $^{\text {b }}$ & $1(<1 \%)$ & $1(<1 \%)$ \\
Cancer in situ $^{\text {b }}$ & $2(<1 \%)$ & $2(1 \%)$ \\
\hline
\end{tabular}

Abbreviations: $\mathrm{DFMO}=$ difluoromethylornithine; $\mathrm{NOS}=$ not otherwise specified

${ }^{a}$ Values are count (column percentag) for categorical variables and mean (range) for continuous variables.

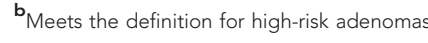

adenoma types were reported: 170 tubular adenomas (77\%), 30 tubulovillous adenomas (13\%), 13 adenoma not otherwise specified (6\%), 6 villous adenomas (3\%), 2 carcinoma in situ $(<1 \%)$ and 1 adenoma with high-grade dysplasia $(<1 \%)$. Adenoma location was reported as follows: rectum $(n=43,19 \%)$, left colon $(n=87,39 \%)$, transverse colon $(31,14 \%)$, right colon $(35,16 \%)$ and cecum (26, 12\%). ODC1 genotype data were available for 157 of 188 end-of-study patients (84\%). ODC1 genotype distribution was as 
follows: GG - 82 patients (52\%), GA - 64 patients (41\%) and AA - 11 patients $(7 \%)$.

Dietary polyamines and selected clinical characteristics at baseline. Across all patients, median total daily intakes of protein (from all sources), animal-derived protein and arginine were 72.8 , 51.8 and $4.0 \mathrm{~g}$, respectively. At baseline, total dietary polyamine intake was correlated with total protein intake $\left(r_{\mathrm{s}}=0.62\right.$, $P<0.0001)$, total animal-derived protein intake $\left(r_{\mathrm{s}}=0.49\right.$, $P<0.0001)$ and total arginine intake $\left(r_{\mathrm{s}}=0.64, P<0.0001\right)$. Dietary arginine intake was highly correlated with the individual dietary polyamine components spermine $\left(r_{\mathrm{s}}=0.90, P<0.0001\right)$ and spermidine $\left(r_{\mathrm{s}}=0.74, P<0.0001\right)$, and to a lesser degree, dietary putrescine $\left(r_{\mathrm{s}}=0.44, P<0.0001\right)$.

The median total daily polyamine intake was $233261 \mathrm{nmol}$ per day (range 48692-740 446). Patients were analysed based on polyamine intake, with the highest (75-100\%) quartile group $v s$ a group including quartiles $1-3(0-25,25-50$ and $50-75 \%)$.

The mean number of adenomas at baseline in the dietary polyamine quartiles $1-3$ was 2.63 ( \pm 2.32 s.d.) compared with 2.39 $( \pm 1.89$ s.d.) in the highest dietary polyamine group, which was not significant $(P=0.86)$. At baseline, the highest dietary polyamine group had a greater proportion of patients with adenomas $>1 \mathrm{~cm}$ (42.9\% vs $26.5 \% ; P=0.022$ ), and as result, a higher proportion with high-risk adenomas $(69.6 \%$ vs $51.8 \% ; P=0.028)$. Left-sided adenomas ( $v s$ right) were more common among patients within dietary polyamine quartiles $1-3(61.5 \%)$ vs the highest dietary polyamine group $(50.0 \%)$, but this difference was not statistically significant (Table 2).

Dietary polyamine intake and rectal tissue polyamines. Twohundred and thirteen patients having rectal tissue polyamine levels were available for analysis. Mean tissue polyamine levels did not significantly differ across the four dietary polyamine quartiles. Total tissue polyamines (nmol polyamines per mg protein) for each dietary polyamine quartile were as follows: quartle $1=11.97$ \pm 5.91 , quartile $2=10.58 \pm 4.93$, quartile $3=10.07 \pm 4.67$ and quartile $4=11.52 \pm 4.47 ; P=0.10)$. When tissue polyamines were analysed individually, tissue levels of putrescine $(P=0.65)$, spermidine $(P=0.15)$, spermine $(P=0.10)$ and the spermidine: spermine ratio $(P=0.51)$ did not significantly differ across the four dietary polyamine groups.

Dietary polyamines and the effect of treatment on adenoma recurrence. Analysis was done on 188 patients with available dietary polyamine intake data and complete trial data with end-ofstudy colonoscopy results to assess effects against adenoma recurrence. In the full logistic regression model, a significant interaction was observed between treatment with DFMO + sulindac and dietary polyamine group with regard to the risk of metachronous adenomas $\left(P_{\text {int }}=0.012\right)$. Similarly, a statistically significant interaction was observed between treatment and dietary polyamine group with regard to risk of metachronous adenomas after further adjustment for quartiles of caloric intake $\left(P_{\text {int }}=0.013\right)$, baseline tissue putrescine $\left(P_{\text {int }}=0.012\right)$, spermidine $\left(P_{\text {int }}=0.014\right)$, spermine $\left(P_{\text {int }}=0.013\right)$ or the spermidine $:$ spermine ratio $\left(P_{\text {int }}=0.010\right)$ into the above risk model. Of note, no significant main effects were observed for energy intake (calories), ODC1 genotype, or any of the baseline tissue polyamine levels on risk of recurrent colorectal adenoma (data not shown).

Table 3 displays the dietary polyamine quartile-specific proportion of patients with colorectal adenoma recurrence after DFMO + sulindac or placebo. In adjusted analysis, we observed a significant $81 \%$ risk reduction of metachronous adenomas from treatment with $\mathrm{DFMO}+$ sulindac ( $v$ s placebo) in the dietary polyamine quartiles 1-3 (43 recurrences, with 7 events among 69 patients in the treatment group and 36 events among 72 patients in the placebo group; risk ratio (RR) 0.19 ; $95 \%$ confidence interval (CI)
Table 2. Baseline adenoma characteristics by dietary polyamine group (baseline cohort; $n=222$ )

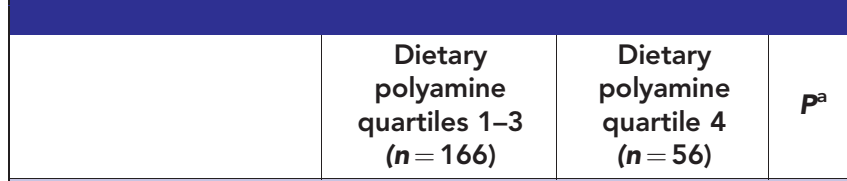

\begin{tabular}{l|l|c|l|}
\hline \multicolumn{4}{|l|}{ Location } \\
\hline Right sided $(n=92)$ & $64(38.6 \%)$ & $28(50.0 \%)$ & 0.13 \\
Left sided $(n=130)$ & $102(61.5 \%)$ & $28(50.0 \%)$ & \\
\hline Mean adenoma number & $2.63( \pm 2.32$ s.d.) & $2.39( \pm 1.89$ s.d.) & 0.86 \\
\hline
\end{tabular}

Number of adenomas

\begin{tabular}{|l|c|c|l|}
\hline 1 & $68(41.0 \%)$ & $25(44.6 \%)$ & 0.16 \\
2 & $44(26.5 \%)$ & $8(14.3 \%)$ & \\
$\geqslant 3$ (multiple adenomas) & $51(30.7 \%)$ & $23(41.1 \%)$ & \\
Missing & $3(1.8 \%)$ & $0(0 \%)$ & \\
\hline $\begin{array}{l}\text { Advanced adenoma } \\
\text { histology }\end{array}$ & $26(15.7 \%)$ & $13(23.2 \%)$ & 0.20 \\
\hline Large adenomas $(\geqslant 1 \mathrm{~cm})^{\mathrm{b}}$ & $44(26.5 \%)$ & $24(42.9 \%)$ & 0.022 \\
\hline High-risk adenomas $^{c}$ & $86(51.8 \%)$ & $39(69.6 \%)$ & 0.020
\end{tabular}

a Wilcoxon rank-sum test $P$-values are reported for the comparison of mean adenoma number; otherwise, $P$-values are reported using $\chi^{2}$-tests for independence. All statistical tests were two-sided.

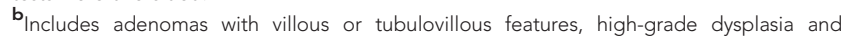
carcinoma in situ.

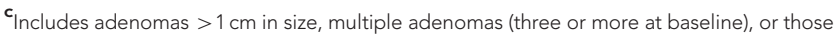
with the following histological characteristics: villous or tubulovillous features, high-grade dysplasia and carcinoma in situ.

$0.08-0.42 ; P<0.0001)$. A non-significant $51 \%$ risk increase was observed for treatment $v s$ placebo in the highest dietary polyamine quartile (11 recurrences, with 5 events among 25 patients in the treatment group and 6 events among 22 patients in the placebo group; RR 1.51; 95\% CI, 0.53-4.29; $P=0.44$; see Supplementary Table 1 for full regression models). In dietary polyamine quartiles $1-3$, significant risk reduction was observed for DFMO + sulindac treatment ( $v s$ placebo) with regard to advanced adenoma recurrence (12 recurrences, with one event among 69 patients in the treatment group and 11 events among 72 patients in the placebo group; RR $0.11 ; 95 \%$ CI $0.01-0.82 ; P=0.032$ ), and for the aggregate end point of advanced adenomas and/or multiple adenomas (17 recurrences, with 1 event among 69 patients in the treatment group and 16 events among 72 patients in the placebo group; RR 0.07; 95\% CI 0.009-0.50; $P=0.009$ ).

\section{DISCUSSION}

In this study, we observed a significant interaction between treatment with DFMO + sulindac and dietary polyamine intake on the risk of recurrent adenomas in a colorectal adenoma prevention trial. Patients in the highest quartile of dietary polyamine intake were found to have no significant metachronous adenoma risk reduction after treatment with DFMO + sulindac, in contrast to a significant $81 \%$ risk reduction observed for patients in dietary polyamine intake quartiles $1-3$.

Patients in the highest dietary polyamine intake group had a greater proportion of large adenomas (42.9\% vs 26.5\%; $P=0.020$ ) at baseline, which accounted for the greater proportion of patients with high-risk adenomas (69.6\% vs 51.8\%; $P=0.020)$ (Table 2). The results in Table 2 generally reflect the results of mouse model 


\begin{tabular}{|c|c|c|c|c|c|}
\hline & $\begin{array}{l}\text { All patients: dietary } \\
\text { polyamine quartiles } \\
\begin{array}{c}1,2,3 \text { and } 4 \\
(n=188)\end{array}\end{array}$ & $\begin{array}{l}\text { Dietary polyamine } \\
\text { quartile } 1 \text { (lowest) } \\
\qquad(n=47)\end{array}$ & $\begin{array}{c}\text { Dietary polyamine } \\
\text { quartile } 2 \\
(n=47)\end{array}$ & $\begin{array}{l}\text { Dietary polyamine } \\
\text { quartile } 3 \\
(n=47)\end{array}$ & $\begin{array}{l}\text { Dietary polyamine } \\
\text { quartile } 4 \text { (highest) } \\
\qquad(n=47)\end{array}$ \\
\hline \multicolumn{6}{|l|}{ Adenoma recurrences } \\
\hline DFMO + sulindac $(n=94)$ & $12 / 94(12.8 \%)$ & $3 / 24(12.5 \%)$ & $1 / 24(4.2 \%)$ & $3 / 21(14.3 \%)$ & $5 / 25(20.0 \%)$ \\
\hline Placebo $(n=94)$ & $42 / 94(44.7 \%)$ & $11 / 23(47.8 \%)$ & $11 / 23(47.8 \%)$ & $14 / 26(53.9 \%)$ & $6 / 22(27.3 \%)$ \\
\hline$P$-value & $<0.0001$ & 0.008 & 0.0006 & 0.005 & 0.56 \\
\hline
\end{tabular}

studies where increased dietary polyamine intake is significantly associated with increasing grade of intestinal adenoma dysplasia (Thomas and Thomas, 2003). In another animal study, it was observed that decreasing polyamine absorption by dietary administration of Bifidobacterium longum resulted in significant suppression of colon tumour incidence, tumour multiplicity and reduction of tumour size by inhibiting the cell proliferation rate as well as ODC activity (Singh et al, 1997).

Dietary polyamine intake did not correlate with tissue polyamine levels in this study. In contrast, experimental studies done by Ignatenko et al (2006) have demonstrated that dietary putrescine supplementation increases intestinal tissue polyamine (e.g., putrescine) levels in $\mathrm{APC}^{\mathrm{Min} /+}$ mice. We propose several theories to explain the tissue polyamine results observed in our study. The free polyamine pool represents only a small portion of the total tissue polyamine pool, and there is no way to measure free polyamines. Because of rapid changes in synthesis, uptake and efflux, it has been proposed that free polyamines induce physiological changes without greatly affecting the larger tissue polyamine pool (Pegg, 2009). Of note, only $10 \%$ of dietary putrescine is retained in body tissues as opposed to $40 \%$ of dietary spermidine (Hughes et al, 1998). In vivo murine models indicate that gastrointestinal tract polyamine transport is mediated by endocytosis and solute carrier transport mechanisms (Pickhardt and Kim, 2009). Externally obtained polyamines are transported into colon cancer cells from the extracellular spaces, and once inside the cell, exogenous putrescine is rapidly converted into spermidine and spermine (which are interconvertible) (Milovic et al, 1998). In addition, it should be noted that tissues contain negatively charged membranes that can act as ion exchange matrixes, and the positively charged polyamines may bind to these negatively charged membranes regardless of polyamine concentration. The current study design and limited sample size may not be optimal to define the complex relationships between dietary polyamine intake and tissue polyamine levels.

Our primary aim was to evaluate the interaction of dietary polyamine intake and treatment with $\mathrm{DFMO}+$ sulindac on metachronous adenoma risk reduction, and a statistically significant interaction was observed $(P=0.012)$. In the highest dietary polyamine group, a non-significant increased recurrence risk was observed after treatment with $\mathrm{DFMO}+$ sulindac $v$ s placebo (RR $1.51 ; 95 \%$ CI 0.53-4.29). In murine experimental studies, inhibition of ODC, induction of spermidine/spermine N1-acetyltransferase and induction of polyamine export by NSAIDs contributed to decreased intracellular polyamine content and apoptosis. Addition of spermidine to the cells prevents apoptosis and restores cell number, suggesting that exogenous polyamines could reverse the efficacy of NSAIDs as observed in our study (Hughes et al, 2003; Thomas and Thomas, 2003). Our data reinforce results from murine experiments, showing that exogenous polyamines govern polyamine homeostasis as well as influence the efficacy of DFMO and sulindac. The reduction in risk of adenoma recurrence from $\mathrm{DFMO}+$ sulindac $v s$ placebo in the dietary polyamine intake quartiles 1-3 (i.e., 3/4ths of the study population) supports ODC inhibition as a promising approach for therapeutic prevention of colon cancer.

Our findings are supported by the experimental work of others reporting on the effects of dietary polyamine depletion in animal models and in humans. In tumour-bearing animal models, investigators studied polyamine deprivation and demonstrated that a polyamine-deficient diet (PDD) significantly enhances the antitumoural effect of DFMO + neomycin (Seiler et al, 1990). Interestingly, in the study treatment of tumour-grafted animals with PDD and neomycin in the drinking water (i.e., polyamine depletion without DFMO administration) resulted in a $40 \%$ inhibition of tumour progression and metastasis. Similarly, chemotherapy effects on tumours were enhanced by PDD, polyamine oxidase inhibition and neomycin (Quemener et al, 1992). Follow-up human studies incorporating a polyaminelowering intervention were then performed. A polyamine-reduced diet combined with partial intermittent intestinal tract decontamination (involving neomycin or nifuroxazide) was investigated in prostate cancer patients in combination with standard therapies over short-term (Cipolla et al, 2003) or long-term durations (Cipolla et al, 2007, 2010). The vast majority of patients in these studies were hormone-refractory prostate cancer patients, who had advanced disease. Nevertheless, the authors demonstrated that the polyamine-lowering intervention was well tolerated and feasible over a prolonged duration (years). The polyamine-lowering regimen was also associated with improved pain control and improved survival characteristics - however, these latter results must be interpreted with caution, as they are not derived from a randomised controlled trial.

Other sources of polyamine regulation should be considered. Genetic polymorphism in ODC1 is associated with CRC survival (Zell et al, 2009b), and may influence the adenoma recurrence risk after treatment with DFMO + sulindac (Zell et al, 2010a). Interaction between $O D C 1$ genotype and dietary polyamine intake may therefore affect polyamine homeostasis. With limited data available in this report, the observed interaction between treatment group and dietary polyamine intake group persisted after additional adjustment for ODC1 genotype.

Here we utilised the previously established dietary polyamine database (Zoumas-Morse et al, 2007) but could not demonstrate a clear relationship between dietary polyamine intake and tissue polyamine levels at baseline. Polyamine content is high in several food products, including certain fruits, nuts, certain cheeses and meat. Our findings are generally congruent with the results from a 
cohort of female CRC cases demonstrating that previously reported NSAID-related mortality risk reduction (Zell et al, 2009a) was restricted to CRC cases reporting the lowest tertile of meat consumption before diagnosis (Zell et al, 2010b). Although meat consumption may be a surrogate for polyamine consumption, substantial amounts of dietary polyamines are also obtained from various foods, such as orange juice, corn and peas. The observed association for total polyamine intake and total daily arginine $\left(r_{\mathrm{s}}=0.64\right)$ or total daily protein intake $\left(r_{\mathrm{s}}=0.62\right)$ was greater than that observed for animal-derived protein intake $\left(r_{s}=0.49\right)$. This relationship between dietary arginine and polyamines supports prior research in $\mathrm{APC}^{\mathrm{Min} /+}$ mice showing that dietary arginineinduced intestinal tumorigenesis can be inhibited by polyamineinhibitory agents such as DFMO and NSAIDs (Zell et al, 2007).

The main limitation of this study is the relatively small sample size $(n=188)$ in the analysis of recurrence risk. With such small numbers, our study did not have sufficient power to perform detailed subset analyses, especially in the highest dietary polyamine group. The observed interaction is derived from multivariate models based on one degree of freedom, whereas the univariate differences based on each dietary polyamine quartile demonstrate modest effects by comparison. Also, it must be acknowledged that the original clinical trial design did not include prospective stratification based on dietary polyamine intake. It is acknowledged that the dietary habits of people can change with time, which could influence our results - especially, given that our analysis is based on the baseline dietary questionnaire and not at various time intervals during the study. However, it is worth noting that there is presumed to be a low likelihood of major dietary changes among middle-aged to older adults during the 3-year on-study in the absence of a life-threatening new medical condition, as patients in this age group typically have a stable and consistent eating pattern. Therefore, using data from the single initial FFQ in the analysis has validity. All dietary-assessment methodologies based on self-report have limitations in accuracy and at best can separate high intake from low intake (as analysed in this study), rather than providing precise intake measurements. Nearly $50 \%$ of patients were taking low-dose aspirin, which could modulate the polyamine body pool; however, the final estimates were adjusted for aspirin use and we did not observe a significant interaction between baseline dietary polyamine intake and aspirin in the logistic regression model (data not shown). Any effects of dietary polyamine intake on the individual contributions of eflornithine or sulindac cannot be determined from these data, as these agents were given in combination vs placebo. Finally, it must be emphasised that our findings are specific to dietary polyamine intake in the setting of this phase III clinical trial of adenoma patients, and do not extend to primary prevention (i.e., potential effects of dietary polyamine intake on CRA or CRC incidence) or to CRC mortality.

In the parent study, a prospective, randomised, placebocontrolled clinical trial of combination of DFMO (a selective inhibitor of polyamine synthesis) and sulindac (a NSAID) over a 3year treatment duration resulted in a $70 \%$ reduction in recurrence of all metachronous adenomas and $>90 \%$ reduction in recurrence of advanced and multiple adenomas compared with placebo. The findings presented here, demonstrating an $81 \%$ risk reduction in metachronous adenomas and $>90 \%$ reduction in advanced adenomas among patients reporting less than the 75th percentile of dietary polyamine intake, suggest that controlling exogenous polyamines could be an adjunctive strategy to therapeutic prevention using polyamine-inhibitory agents. This may prove to be important in the future, as secondary and tertiary CRC prevention trials utilising polyamine-inhibitory agents are developed. However, the effect of dietary polyamine intake on tissue polyamine levels in humans is complex. Our results are confounded by a lower number of recurrent events in the placebo group within dietary polyamine group 4 , and also we did not observe the expected linear relationship between dietary and tissue polyamines in this study. Thus, the findings reported here are considered to be exploratory, and warrant investigation in future research - particularly clinical trial-based research where the dietary intervention is controlled.

\section{ACKNOWLEDGEMENTS}

This study was supported by the National Cancer Institute contract no. NO1-CN75019 (FLM and CEM) and grants CA59024 and CA62230 (to FLM); CA88078 (to FLM and CEM); and CA47396, CA72008 and CA95060 (to EWG). We thank the participating institutions and investigators: Chao Family Comprehensive Cancer Center, University of California Irvine, CA: Frank L Meyskens Jr, MD (Principal Investigator); Gregory Albers, MD; Sharon Fujikawa-Brooks, PhD; Philip M. Carpenter, MD; Daniel L. Gillen, PhD; Christine E McLaren, PhD; Daniel Pelot, MD Arizona Cancer Center, University of Arizona, AZ: Eugene W Gerner, PhD; Steven Goldschmid, MD; Peter Lance, MD. Denver Department of Veteran Affairs Medical Center and University of Colorado: Dennis J Ahnen, MD. Department of Veterans Affairs Long Beach Healthcare System, CA: Jayashri Kidao, MD. Kaiser Permanente, Sacramento, CA: Michael J Lawson, MD. Loma Linda University, CA: John McCracken, MD. Department of Veterans Affairs Loma Linda Healthcare System, CA: Ronald Griffen, MD. University of Michigan, Ann Arbor, CA: D. Kim Turgeon, MD. University of Kansas, Kansas City, CA: Curt H Hagedorn, MD.

\section{CONFLICT OF INTEREST}

FLM and EWG have ownership interest in Cancer Prevention Pharmaceuticals, LLC. The remaining authors declare no conflict of interest.

\section{REFERENCES}

Bachrach U, Wang YC, Tabib A (2001) Polyamines: new cues in cellular signal transduction. News Physiol Sci 16: 106-109.

Boyle JO, Meyskens Jr. FL, Garewal HS, Gerner EW (1992) Polyamine contents in rectal and buccal mucosae in humans treated with oral difluoromethylornithine. Cancer Epidemiol Biomarkers Prev 1(2): 131-135.

Brabender J, Lord RV, Danenberg KD, Metzger R, Schneider PM, Uetake H, Kawakami K, Park JM, Salonga D, Peters JH, DeMeester TR, Holscher AH, Danenberg PV (2001) Upregulation of ornithine decarboxylase mRNA expression in Barrett's esophagus and Barrett's-associated adenocarcinoma. J Gastrointest Surg 5(2): 174-181, discussion 182.

Cipolla B, Guilli F, Moulinoux JP (2003) Polyamine-reduced diet in metastatic hormone-refractory prostate cancer (HRPC) patients. Biochem Soc Trans 31(2): 384-387.

Cipolla BG, Havouis R, Moulinoux JP (2007) Polyamine contents in current foods: a basis for polyamine reduced diet and a study of its long term observance and tolerance in prostate carcinoma patients. Amino Acids 33(2): 203-212.

Cipolla BG, Havouis R, Moulinoux JP (2010) Polyamine reduced diet (PRD) nutrition therapy in hormone refractory prostate cancer patients. Biomed Pharmacother 64(5): 363-368.

Fearon ER, Vogelstein B (1990) A genetic model for colorectal tumorigenesis. Cell 61(5): 759-767.

Gerner EW, Meyskens Jr FL (2004) Polyamines and cancer: old molecules, new understanding. Nat Rev Cancer 4(10): 781-792.

Guo YJ, Harris RB, Rosson D, Boorman D, O’Brien TG (2000) Functional analysis of human ornithine decarboxylase alleles. Cancer Res 60(22): 6314-6317. 
Hughes A, Smith NI, Wallace HM (2003) Polyamines reverse non-steroidal anti-inflammatory drug-induced toxicity in human colorectal cancer cells. Biochem J 374(Pt 2): 481-488.

Hughes EL, Grant G, Pusztai A, Bardocz S (1998) Uptake and interorgan distribution of dietary polyamines in the rat. Biochem Soc Trans 26(4): S369.

Ignatenko NA, Besselsen DG, Roy UK, Stringer DE, Blohm-Mangone KA, Padilla-Torres JL, Guillen RJ, Gerner EW (2006) Dietary putrescine reduces the intestinal anticarcinogenic activity of sulindac in a murine model of familial adenomatous polyposis. Nutr Cancer 56(2): $172-181$.

Jass JR, Whitehall VL, Young J, Leggett BA (2002) Emerging concepts in colorectal neoplasia. Gastroenterology 123(3): 862-876.

Kristal AR, Shattuck AL, Williams AE (1994) Food frequency questionnaires for diet intervention research. Proceedings of the 17th National Nutrient Databank Conference; Baltimore, MD, 7-10 June, 1992. International Life Sciences Institute: Washington, DC, pp 110-125.

Linsalata M, Russo F (2008) Nutritional factors and polyamine metabolism in colorectal cancer. Nutrition 24(4): 382-389.

McGarrity TJ, Peiffer LP, Bartholomew MJ, Pegg AE (1990) Colonic polyamine content and ornithine decarboxylase activity as markers for adenomas. Cancer 66(7): 1539-1543.

Meyskens Jr FL, Gerner EW (1999) Development of difluoromethylornithine (DFMO) as a chemoprevention agent. Clin Cancer Res 5(5): 945-951.

Meyskens FL, Gerner EW, Emerson S, Pelot D, Durbin T, Doyle K, Lagerberg W (1998) Effect of alpha-difluoromethylornithine on rectal mucosal levels of polyamines in a randomized, double-blinded trial for colon cancer prevention. J Natl Cancer Inst 90(16): 1212-1218.

Meyskens Jr FL, McLaren CE, Pelot D, Fujikawa-Brooks S, Carpenter PM, Hawk E, Kelloff G, Lawson MJ, Kidao J, McCracken J, Albers CG, Ahnen DJ, Turgeon DK, Goldschmid S, Lance P, Hagedorn CH, Gillen DL, Gerner EW (2008) Difluoromethylornithine plus sulindac for the prevention of sporadic colorectal adenomas: a randomized placebo-controlled, double-blind trial. Cancer Prev Res 1(1): 32-38.

Milovic V, Turhanowa L, Fares FA, Lerner A, Caspary WF, Stein J (1998) S-adenosylmethionine decarboxylase activity and utilization of exogenous putrescine are enhanced in colon cancer cells stimulated to grow by EGF. $Z$ Gastroenterol 36(11): 947-954.

Pegg AE (2009) Mammalian polyamine metabolism and function. IUBMB Life 61(9): $880-894$.

Pickhardt PJ, Kim DH (2009) Advanced vs 'high-risk' adenomas: identifying the relevant target for CT colonography screening. Am J Gastroenterol 104(6): 1599-1600, author reply 1600.

Quemener V, Moulinoux JP, Havouis R, Seiler N (1992) Polyamine deprivation enhances antitumoral efficacy of chemotherapy. Anticancer Res 12(5): 1447-1453.

Schakel SF, Buzzard IM, Gebhardt SE (1997) Procedures for estimating nutrient values for food composition databases. J Food Comp Anal 10: $102-114$.

Seiler N, Knodgen B (1980) High-performance liquid chromatographic procedure for the simultaneous determination of the natural polyamines and their monoacetyl derivatives. J Chromatogr 221(2): 227-235.
Seiler N, Sarhan S, Grauffel C, Jones R, Knodgen B, Moulinoux JP (1990) Endogenous and exogenous polyamines in support of tumor growth. Cancer Res 50(16): 5077-5083.

Siegel R, Ward E, Brawley O, Jemal A (2011) Cancer statistics, 2011: the impact of eliminating socioeconomic and racial disparities on premature cancer deaths. CA Cancer J Clin 61(4): 212-236.

Singh J, Rivenson A, Tomita M, Shimamura S, Ishibashi N, Reddy BS (1997) Bifidobacterium longum, a lactic acid-producing intestinal bacterium inhibits colon cancer and modulates the intermediate biomarkers of colon carcinogenesis. Carcinogenesis 18(4): 833-841.

Tabib A, Bachrach U (1999) Role of polyamines in mediating malignant transformation and oncogene expression. Int J Biochem Cell Biol 31(11): 1289-1295.

Thomas T, Thomas TJ (2003) Polyamine metabolism and cancer. J Cell Mol Med 7(2): 113-126.

Uemura T, Stringer DE, Blohm-Mangone KA, Gerner EW (2010) Polyamine transport is mediated by both endocytic and solute carrier transport mechanisms in the gastrointestinal tract. Am J Physiol Gastrointest Liver Physiol 299(2): G517-G522.

Wallace HM (1996) Polyamines in human health. Proc Nutr Soc 55(1B): 419-431.

Wang W, Liu LQ, Higuchi CM (1996) Mucosal polyamine measurements and colorectal cancer risk. J Cell Biochem 63(2): 252-257.

Zell JA, Ignatenko NA, Yerushalmi HF, Ziogas A, Besselsen DG, Gerner EW, Anton-Culver H (2007) Risk and risk reduction involving arginine intake and meat consumption in colorectal tumorigenesis and survival. Int $J$ Cancer 120(3): 459-468.

Zell JA, McLaren CE, Chen WP, Thompson PA, Gerner EW, Meyskens FL (2010a) Ornithine decarboxylase-1 polymorphism, chemoprevention with eflornithine and sulindac, and outcomes among colorectal adenoma patients. J Natl Cancer Inst 102(19): 1513-1516.

Zell JA, Ziogas A, Bernstein L, Clarke CA, Deapen D, Largent JA, Neuhausen SL, Stram DO, Ursin G, Anton-Culver H (2009a) Nonsteroidal antiinflammatory drugs: effects on mortality after colorectal cancer diagnosis. Cancer 115(24): 5662-5671.

Zell JA, Ziogas A, Bernstein L, Clarke CA, Deapen D, Largent JA, Neuhausen SL, Stram DO, Ursin G, Anton-Culver H (2010b) Meat consumption, nonsteroidal anti-inflammatory drug use, and mortality among colorectal cancer patients in the california teachers study. Cancer Prev Res (Phila Pa) 3(7): 865-875.

Zell JA, Ziogas A, Ignatenko N, Honda J, Qu N, Bobbs AS, Neuhausen SL, Gerner EW, Anton-Culver H (2009b) Associations of a polymorphism in the ornithine decarboxylase gene with colorectal cancer survival. Clin Cancer Res 15(19): 6208-6216.

Zoumas-Morse C, Rock CL, Quintana EL, Neuhouser ML, Gerner EW, Meyskens Jr FL (2007) Development of a polyamine database for assessing dietary intake. J Am Diet Assoc 107(6): 1024-1027.

This work is published under the standard license to publish agreement. After 12 months the work will become freely available and the license terms will switch to a Creative Commons AttributionNonCommercial-Share Alike 3.0 Unported License.

Supplementary Information accompanies this paper on British Journal of Cancer website (http://www.nature.com/bjc) 